\title{
Le musée d'histoire en France entre traditions nationales et soucis identitaires
}

\begin{abstract}
Dominique Poulot ${ }^{1}$
RÉSUMÉ: Les musées d'histoire ont connu durant les dernières décennies en France des mutations rapides, marquées par une série de constructions ou de rénovations. L'histoire de tels chantiers, intervenus plus tardivement par rapport aux autres types de musées, permet de s'interroger sur les rapports nouveaux tissés à ces occasions entre musées d'histoire et historiographie savante. On constate que le musée d'histoire, aujourd'hui, est davantage un musée du présent qu'un musée érudit au sens traditionnel du terme. Refusant la nostalgie, le musée idéal doit élaborer une nouvelle représentation du patrimoine conçue comme une dynamique inédite du " patrimoine » dans la société. L'enjeu est de refuser une marchandisation spectaculaire de I'histoire qui exclut l'approche critique et savante, tout en combattant l'éventuelle disparition de la spécificité muséographique derrière le seul savoir historien, aux dépens des revendications de la mémoire sociale. Dans ces conditions, le rapport du patrimoine et de l'écriture de I'histoire devient un enjeu central pour les musées d'histoire.

MOTS CLÉS: Musée. Patrimoine. Historiographie. Philosophie de l'histoire. Nationalisme.

RESUMO: Os museus de história na França conheceram, durante as últimas décadas, rápidas mudanças marcadas por uma série de construções ou reformas. A história desses canteiros de obra, que se deu mais tarde em comparação às intervenções em outros tipos de museu, permitenos questionar as novas relações tecidas, nessas ocasiões, entre os museus de história e a historiografia erudita. Constata-se que o museu de história é hoje mais um museu do presente do que um museu erudito, no sentido tradicional do termo. Sem se prender à nostalgia, o museu ideal deve elaborar uma nova representação do ?patrimônio?, concebendo-o como uma dinâmica inédita dentro da sociedade. $\bigcirc$ desafio consiste em recusar uma ? mercantilização? espetacular da história que exclua a abordagem crítica e erudita, combatendo, ao mesmo tempo, o possível desaparecimento da especificidade museográfica por trás do raro saber histórico à custa das reivindicações da memória social. Nessas condições, a relação entre o patrimônio e a historiografia torna-se o principal desafio para os museus de história. PAlaVRas-CHAVE: Museu. Patrimônio. Historiografia. Filosofia da História. Nacionalismo.
\end{abstract}

1. Université Paris I - Panthéon-Sorbonne, Laboratoire d'Anthropologie et d'Histoire de l'Institution de la Culture, Centre National de la Recherche ScientifiqueEcole des Hautes Etudes en Sciences Sociales. E-mail $<$ dominique.poulot@univ-pa ris1.fr $>$ 
2. Ceci dans la ligne de l'article séminal de Roland Barthes (1967). Voir en particulier S. Bann (1984). Et sur l'oeil de l'historien Jo Tollebeek (2000), ainsi que pour une comparaison avec le musée d'ethnographie N. Dias (1994). Sur le thème de Michelet qui voit les monuments (au musée) et de Thierry qui lit les documents, cf. les remarques de Marcel Gauchet (1986) et de François Hartog (1996)

3. Je renvoie à Paul Ricoeur (2000, p. 746-747)

4. On se reportera aux réflexions de J. Le Goff (1977) et à sa préface à Marc Bloch (LE GOFF, 1997).Vue d'ensemble contemporaine du cadre professionnel dans Gaynor Kavanagh (1990).
ABSTRACT: The museums of history have known some spectacular changes in contemporary France for two decades. New buildings have been constructed, and a lot of old ones have been renovated. The study of some specific cases, for example the museums devoted to the history of World War II, or to the history of migrations, demonstrates how museology is nowadays far from the pure illustration of erudite knowledge. The ideal museum of french history is today a museum very concerned by the present state of affairs, the political agenda or the social agency. Its aims are linked to the consciousness of « heritage » in the contemporary society. One difficulty is not to becoming a commercial product, a kind of entertainment, or of " edutainment " for kids. The challenge is to play the role of a place of memory, or even to figure a tool for collective thinking about the new stakes of national identity.

KEYWORDS: Museum. Heritage. Historiography. Philosophy of History. Nationalism.

Les musées d'histoire connaissent en France des mutations rapides, marquées par une série de constructions ou de rénovations, et l'annonce de l'ouverture de différents chantiers. Cette modernisation générale de l'institution est intervenue après celle des musées d'art. Le phénomène vaut d'être noté, car le considérable succès de l'école historique française des années 1960-1980 n'avait pas débouché sur un renouveau des expositions ou des musées d'histoire. Les plus éminents des historiens avaient alors investi l'édition, ou la télévision, bien davantage qu'ils n'avaient participé à des entreprises muséales, voire plus largement patrimoniales. Pareille observation pose donc d'entrée de jeu la question du rapport entre musées d'histoire et vicissitudes historiographiques.

Le rapport entre musées d'histoire et historiographie relève a priori de l'évidence. Car les musées d'histoire ne sont pas - ou pas seulement-des musées exposant des collections historiques: auquel cas tout musée serait a priori un musée d'histoire. Ce sont des musées qui se nourrissent et se réclament de l'histoire, c'est-à-dire du regard et du travail des historiens professionnels - universitaires pour l'essentiel. De ce point de vue, on ne discerne aucune règle précise quant aux rapports entretenus par tel ou tel établissement avec le monde savant - ainsi d'éventuels critères d'organisation, de taille ou de qualité de la collection, de financement, de règles de gestion et de direction etc., qui amèneraient un musée à se soucier davantage qu'un autre de régler ses expositions sur le savoir académique. Autrement dit, les musées d'histoire varient de manière considérable quant à leurs objectifs et à leurs obligations par rapport à ce qu'il est convenu d'appeler la science historique. Enfin, la diversité des muséographies d'histoire ne peut être sous-estimée. Certains musées donnent à voir l'unité de l'histoire, voire sa clôture, à partir des matériaux rassemblés². Mais d'autres établissements illustrent la représentation du passé sur le mode de ce "petit bonheur, le petit miracle de la reconnaissance et de son moment d'intuition et de croyance immédiate", "la reconnaissance du passé comme ayant été quoique n'étant plus" 3 .

De manière générale, le musée d'histoire travaille moins au répertoire des sources de l'histoire qu'il ne sanctionne l'émergence de curiosités nouvelles, en pesant sur les vicissitudes des intérêts savants, en vulgarisant (plus ou moins bien) les acquis érudits auprès des visiteurs, par le biais de catalogues et de notices ${ }^{4}$. Mais si le lien avec les archives est clair dans la constitution d'une histoire savante, les révolutions successives de l'écriture de l'histoire engageant systématiquement ou presque des révolutions documentaires, il n'en va de même pour les collections 
des musées d'histoire, et pour leurs modes d'interprétation. L'éventuelle interaction entre la représentation vulgarisatrice des acquis de la recherche dans les musées et l'effet, en retour, des représentations muséographiques sur l'historiographie savante n'a guère été abordée.

Une question mal posée: des historiens flattés et frustrés

Les historiens français ne se sont guère intéressés à l'histoire des musées avant le début des années 1990: les grands colloques datent du milieu de la décennie, liés à des commémorations - tel le colloque organisé par le Service culturel du musée du Louvre à l'occasion de la commémoration du bicentenaire du musée les 3, 4 et 5 juin 1993 - ou à des expositions à l'intérieur même des musées $^{5}$. L'intérêt des historiens de l'art, pour être bien antérieur, n'en était pas moins limité aux relations entre artistes et institutions - quand il n'était pas exclusivement focalisé sur l'histoire des décors de ces établissements ${ }^{6}$.

D'une manière plus spécifique, nombre d'historiens universitaires ont développé une forme de célébration, des musées d'histoire, qu'ils aient été ou non partie prenante dans leur réalisation, dans la mesure où ces établissements leur semblent - à plus ou moins juste titre - autant de tribunes ou d'outils afin d'affirmer un engagement civique, une utilité sociale, une autorité intellectuelle de l'histoire. Pour d'autres, cependant, l'analyse du musée d'histoire est surtout prétexte à se livrer à un retour critique sur le rapport qu'entretient la fiction muséographique - avec le travail érudit; pareille réflexion s'inspire souvent des travaux de la génération des années 1970 (Michel de Certeau), à partir des perspectives sémiotiques ouvertes tant par la mise en évidence de "l'effet de réel" (Roland Barthes) que par la poétique des tropes au sein d'une "métahistoire" (Hayden White). Enfin une critique volontiers radicale des pouvoirs du musée, sur le mode de la critique du spectacle, développe une condamnation plus générale du musée d'histoire contemporain, qui semble condamné à sacrifier tant aux dispositifs de la consommation touristique qu'à un devoir conventionnel de mémoire. Cette perspective a été particulièrement développée à propos de deux musées, consacrés respectivement à la première et à la seconde guerre mondiale, celui de Péronne et celui de Caen. A Péronne, le débat se complique de polémiques ou de conflits d'interprétation entre ce qu'il est convenu d'appeler "I'école de Péronne", et ses critiques qui, tels Nicolas Offenstadt ou Rémy Cazals, soutiennent que ce ne sont pas nos sentiments humanistes d'aujourd'hui qui survalorisent les mutineries et leur répression, mais qu'il s'agit bien là de sentiments contemporains, ce dont la lecture des témoignages d'époque - les journaux des tranchées - doit nous convaincre.

Reste que le musée est majoritairement abordé par les historiens sur le mode sinon du mépris, au moins de la condescendance, le cas échéant au nom d'un appel à son gouvernement par les historiens eux-mêmes. A quoi répond un discours non moins stéréotypé des conservateurs et autres professionnels des établissements quant à la spécificité du métier et quant aux exigences propres du media qu'est
5. La jeunesse des musées... (1994); Les musées en Europe... (1995); voir aussi M.-H. Joly et $\mathrm{T}$. Compère-Morel (1998).

6. La tradition d'histoire des musées, dans la perspective d'une histoire culturelle de l'art, a été ouverte par les travaux de Pierre Angrand (1984-1988), Thomas W. Gaehtgens (1984; 1986), Marie-Claude Chaudonneret (1991; 2004), et Sébastien Allard (2006), qui développe l'essai synthétique de Pierre Vaisse dans le catalogue $L a$ jeunesse des musées, op. cit.

7.“[...] l'historial de Péronne est un lieu pour écrire une histoire qui juge et qui condamne la conflit et qui travaille à son effacement.A ce titre l'historial est le lieu de l'invention d'une mémoire européenne pour le présent [...] Caen laisse peu de place à la mémoire de la seconde guerre mondiale. Il en propose surtout une histoire très construite [...] Pour le temps présent, il juxtapose à cete histoire des discours idéologiques qui ne sont pas à proprement parler une élaboration de la mémoire de la guerre, et des mémoriaux qui à l'échelle du débarquement et de la bataille de Normandie ne sont que des évocations de ce qu'il faudra bien aller chercher ailleurs" (WAHNICH, 2005, p. 80-81). 
8. Le Débat, 44, 1987. Voir le bilan fourni sur ce débat par Daniel J. Sherman (1990).

9. Le programme d'un grand musée d'histoire, évoqué par différents historiens et conservateurs au cours de la dernière décennie au $\mathrm{XX}^{\text {ème }}$ siècle, n'a aucune chance d'aboutir aujourd'hui que le Mucem, Musée des civilisations de l'Europe et de la Méditerranée, est en voie d'achèvement à Marseille, nouvel avatar du musée des Arts etTraditions populaires.

10. Cf. Boursier (1997)

11. Le Centre d'histoire de la Résistance et de la Déportation a été créé en 1992 à Lyon, symboliquement installé dans l'ancienne école de santé militaire, occupée par la Gestapo. L'exposition permanente est d'immersion: le visiteur, muni d'un audioguide, pénètre dans un univers visuel et sonore de la Seconde Guerre mondiale. le musée. Bref, tout se passe comme si les historiens déploraient de ne pas contrôler, ou de ne plus contrôler, les musées d'histoire, leurs discours et leurs scénographies, et aspiraient à une reconquête, voire à un renouveau d'usages savants perdus. De là une éventuelle censure des dispositifs muséographiques imaginés par des conservateurs tenus pour autant d'historiens amateurs par les historiens académiques. Une manifestation spectaculaire en a été fournie naguère dans le dossier réuni par la revue Le Débat à l'occasion de l'inauguration du musée d'Orsay: Maurice Agulhon y donnait une appréciation de la partie proprement historique du musée, à savoir le "passage des dates", seul témoignage d'une ambition historienne dans ce musée d'art. Il le faisait explicitement à la manière dont un professeur corrige une copie d'élève, distribuant bons et mauvais points, ou encore à la manière dont un professionnel peut considérer ceux qui $s^{\prime} a v e n t u r e n t$ en amateurs sur son terrain ${ }^{8}$. C'était là poser le débat en termes d'alternative entre un musée conservatoire d'objets, ou d'images, et une institution capable d'initier à une histoire spécifique - de la culture matérielle, ou du visuel.

Le regret, devenu banal, de ce qu'il est convenu d'appeler un divorce du musée et des historiens suppose qu'il y avait naguère une heureuse union - ou au moins un mariage de raison - entre historiens et musées, ce qui relève largement de l'invention a posteriori d'un âge d'or perdu, au nom duquel on jugerait aujourd'hui un présent déplorable. En fait, sur la longue durée, la responsabilité de l'histoire dans les musées d'histoire n'a jamais appartenu directement aux historiens. Pour le dire rapidement, en France au moins, les historiens professionnels ne sont généralement pas à l'origine des musées d'histoire. Certes, ils peuvent lancer des pétitions en faveur de la réalisation de tel ou tel établissement idéa ${ }^{9}$ mais de telles initiatives ont tous les risques d'apparaître corporatistes et de ne rencontrer qu'un silence assourdissant. Les initiatives proviennent au cours du XXème siècle d'anciens acteurs de l'histoire - pour la seconde guerre mondiale de déportés, de résistants etc. - , ou de collectionneurs passionnés ${ }^{10}$. La décision de bâtir un musée d'histoire, tout au long de l'histoire contemporaine française, vient généralement du milieu politique, local ou national, et répond à telle ou telle actualité. Ainsi, le procès Barbie, un tortionnaire de la Gestapo, en 1987'1, et la redécouverte de la tragédie des enfants juifs déportés d'Izieu ont-ils conduit à créer la Maison des enfants d'lzieu, et un musée à Lyon, tandis que la commémoration du bicentenaire de la Révolution française a amené le département de l'Isère à ouvrir un musée de la Révolution à Vizille, sans disposer préalablement d'aucune collection.

Une tradition muséographique spécifique

Le XIXème siècle a connu, dès l'origine, des musées d'histoire contemporaine. Telles sont les salles de Napoléon, de l'expédition d'Egypte ou des moulages de monuments commandés par l'Empereur, que Lenoir avait prévu d'intituler salles du XIXème siècle; tel est le musée de Versailles de Lovis-Philippe représentant la Révolution 
et la conquête de l'Afrique du Nord, tel est encore, sur un autre plan, le musée des Souverains sous Napoléon III. Ces musées se répartissent, sommairement, entre des musées consacrés aux annales de la France et à ses grands hommes, des laboratoires de la science /archéologique, historique, paléographique, ethnohistorique), enfin des représentations d'un être collectif, d'une identité maintenue à travers les âges, comme en suspens ou à l'écart des vicissitudes plus communes de I'histoire, sur le mode d'un présent perpétuellement réécrit.

L'émergence neuve d'un culte des grands hommes est à l'évidence un élément essentiel de la société des Lumières qui, à travers ce culte, "n'en finit pas de se raconter à elle-même son propre avènement"12. L'essentiel tient en effet à l'éloge de la valeur des génies pour leur temps et leur nation, éloge qui s'inscrit dans une crise des systèmes traditionnels de représentation. Dans la littérature utopique, particulièrement, commente B. Baczko, le discours historique se confond avec un discours moralisant et didactique - "un discours que l'on pourrait qualifier de 'monumental' en ce sens qu'il est parfaitement traduisible en une série de monuments qui seraient autant d'images distribuant au peuple des exemples édifiants et des leçons de morale"13. Au début du règne de Louis XVI, la France connaît, avec le comte d'Angiviller (1730-1809), nommé à la Direction des Bâtiments, une nouvelle politique de la postérité: I'un des symboles en est, en décembre 1774 et janvier 1775, la commande chaque année de "tableaux d'histoire et de statues dont le sujet sera les grands hommes français"14. La série, d'intention patriotique, obéit à la rhétorique du "moment significatif": chaque sculpture illustre une attitude moralement et historiquement significative du personnage, longuement explicitée dans le livret du Salon. Poursuivie régulièrement de 1776 à 1787 elle donne lieu à vingt-sept statues ${ }^{15}$. Cette politique de l'imagerie nationale s'inscrit dans un débat sur les valeurs à reconnaître à l'histoire française, et sur les éventuels modèles patriotiques à y trouver ${ }^{16}$.

Par la suite, la Révolution semble procurer l'opportunité d'une politique de la mémoire enfin morale et rationnelle. Au musée des Monuments français, I'obligation impérieuse d'intelligence de l'héritage historique requiert un effort de clarté et d'efficacité qui eût été auparavant parfaitement incongru. Au musée de Lenoir, la distribution des œuvres obéit à un classement "par âge et par ordre de date, c'est-à-dire en autant de pièces séparées que l'art nous offre d'époques remarquables". La succession de salles offre le résumé "des monuments de tous les siècles, chronologiquement placés". La spécificité des Petits-Augustins tient à son décor, qui veut donner "à chacune des salles le caractère, la physionomie exacte du siècle qu'elle doit représenter"17, grâce au réemploi de "détails" provenant de monuments détruits, voire dépecés à l'initiative de Lenoir lui-même.

Sous la Restauration le Musée des monuments français est fermé au nom d'une politique de la tradition continuée ${ }^{18}$, et dans une perspective d'expiation ${ }^{19}$. Un nouveau type de musée d'histoire apparaît peu après, au service des annales de la France. Tel est le musée de Louis-Philippe dont Victor Hugo dira à son inauguration triomphale le 10 juin 1837 qu'il "a donné à ce livre magnifique qu'on appelle I'histoire de France cette magnifique reliure qu'on appelle Versailles" 20 . Versailles illustre les vertus du musée de site, pour détourner une expression contemporaine,
12. Cf. Jean-Claude Bonnet (1998). On lira aussi les contributions réunies dans le catalogue d'exposition dirigé par B. Bergdoll (1989), notamment M. K. Deming (1989, p. 97-150).

13. Cf. B. Baczko (1978, p. 322-323).

14. Cf. McClellan (1993).

15. Voir Fr. Souchal (1979, p. 117-121).

16. Sur l'ensemble du phénomène la référence demeure Lionel Gossman (1968), auquel on peut ajouter ses essais (GOSSMAN, 1990).

17. Catalogue du Musée des monuments français, 1810 , p. 6.

18. L'étude de référence demeure l'ouvrage de Stanley Mellon (1955).

19.Avec l'ordonnance du 18 décembre 1816 , le lieu, consacré désormais à l'Ecole des Beaux-arts, commence à recevoir une nouvelle collection, de moulages d'antiques cette fois. Cf. Ch. Pinatel (1992). Parallèlement, l'histoire de la famille royale est représentée sur le mode de l'expiation: voir R. Anchel (1924); J.-M. Darnis (1981); et G. Bresc-Bautier (1981).

20. Cf. Hugo (1972, p. 153 154). Il commente: "Ce que le roi Louis-Philippe a fait à Versailles est bien. Avoir accompli cette oeuvre, c'est avoir été grand comme roi et impartial comme philosophe; c'est avoir fait un monument national d'un monument monarchique; c'est avoir mis une idée immense dans le passé, 1789 vis-à-vis de 1688 , l'empereur chez le roi, Napoléon chez Louis XIV". 
21. Cf. Jean Adhémar (1956, 1981).

22. Un colloque a été organisé au château de Versailles les 10, 11 et 12 décembre 1998 à ce sujet:cf.les actes, en $L$ 'bistoire au musée, 2004.

23. Cf. F. Berçé (1993) et P. Marot (1981).

24. Voir notamment Claudie Bernard (1996), et à propos du Roman de la momie de Gautier (BERNARD, 1991).

25. J'emprunte le concept à M. Bakhtine (1978).

26. Le catalogue analytique Les arts au Moyen Age, (infol.et $5 v$.de texte, 1842-1846) annonce les musées d'arts décoratifs européens

27. Cf. S. Bann (1984, p. 60 61). Rappelons que Jakobson fait de la prédominance de la métonymie et de la synecdoque la caractéristique du réalisme, par opposition à la métaphore, qui définit romantisme et symbolisme: ces relations de contiguité permettent de passer des personnages à leur contexte, de l'intrigue à l'atmosphère (JAKOBSON, 1973).

28. Sur Boucher de Perthes voir Cl. Cohen et J. J. Hublin (1989) et Eve Gran-Aymerich (1998).

29. Cf. Aux grands... (2004).

30. Voir Nathalie Richard (1991). tant le château était un lieu à (ré)occuper: un lieu dont il fallait inventer la destination à nouveaux frais. Le projet de Louis-Philippe retrouve, comme l'a relevé Jean Adhémar, la tradition de l'enseignement du Prince et de l'usage pédagogique des images, dans une longue durée des galeries de portraits et des histoires de France qui voit rééditer Anquetil et l'abbé Velly, et place toujours le légendaire Pharamond au seuil de la France ${ }^{21}$. Le musée de Versailles acculture en France l'idée de musée d'histoire: il en fait respecter le principe, après une première réalisation, celle de Lenoir, à la fois trop idéologique et trop personnelle, bref marginale. Avec Versailles, le musée d'histoire acquiert définitivement une légitimitée 22 .

L'élaboration du Musée des thermes de Cluny sous l'autorité de la Commission des Monuments historiques, créée en $1837^{23}$, inaugure une culture des lieux au sein des musées d'histoire. Les installations suggestives conçue par Alexandre du Sommerard (1779-1842) - ainsi dans la chambre de François ler - s'inscrivent explicitement dans une quête d'expériences du passé que le triomphe du roman historique a largement popularisée ${ }^{24}$, donnant lieu à un véritable "chronotope" 25 du Moyen-Age et de la Renaissance. A la manière de l'école narrative imaginée par Prosper de Barante, où les sources originales donnent la parole au passé comme pour se raconter lui-même, la mise en scène de reliques et de pièces d'art décoratif fournit une expérience sensible de la différence des temps ${ }^{26}$. Chez Lenoir, l'esprit d'un siècle s'exprimait dans les extraits de "monuments" disposés afin de représenter l'état de chaque siècle; chez Du Sommerard les "antiquités" contribuent à recréer différents contextes ou milieux, qui évoquent des usages et des pratiques, et restituent un cadre de vie ${ }^{27}$.

Un autre type de musée d'histoire est l'atelier, consacré à l'avenir de la science davantage qu'à la présence d'un passé ressuscité ou qu'à l'impératif de transmission à la postérité. Il s'agit de travailler à l'élaboration de l'histoire à partir de reliques ou de traces trouvées le plus souvent in situ. En 1835 Boucher de Perthes propose ainsi de fonder différents musées d'antiquités françaises, galloromaines, gauloises et celtiques ${ }^{28}$ : "ce n'était pas la nature même du dépôt et sa valeur métallique, ou sa beauté réelle, qui en aurait fait le prix, prévientill, mais les conséquences qu'on eût pu en déduire. En un mot, c'eût été un cadre pour l'avenir". Le musée des archives, ouvert en 1867 se veut de même une école de travaux pratiques de la science, à l'inverse du musée de Versailles, qui fait figure de véritable repoussoir ${ }^{29}$. Comme l'affirme la Revue des Questions Historiques "c'est devant ces monuments du passé qu'il ferait bon professer un cours de paléographie élémentaire". Le règne de Napoléon III voit encore la fondation du musée des antiquités nationales en 1862 au château de Saint-Germain, sous l'autorité d'une commission de savants. Là aussi une sociabilité savante s'organise autour du musée et alimente la jeune recherche préhistorique ${ }^{30}$.

Les premiers musées d'histoire, issus de la Révolution, héritiers d'une mentalité discriminatoire propre aux utopies éclairées, avaient incarné une toute-puissance de l'institution devant le passé défunt. C'est au musée, installé dans une temporalité neuve, que le passé pouvait acquérir une signification dont il était cruellement dépourvu en tant que tel. Sous la monarchie de juillet, une conception annaliste de l'histoire confie la surveillance de son écriture et de sa transmission au Prince 
et à ses conseillers. Le musée renoue les fils de l'ancienne et de la nouvelle France, grâce à une mise en scène prestigieuse, au sein d'un cadre patrimonial. Au milieu du XIXème siècle, et une fois oublié l'imaginaire du temps refait à neuf, un dessein de musée atelier apparaît, lié à un idéal laborieux, quasi archivistique, de rapport à l'histoire: il appelle le travail savant comme les efforts d'une sociabilité érudite.

Par la suite, la vie du peuple à travers l'histoire occupe une place grandissante dans la mise en scène des musées ou des expositions : c'est de "nous", de l'histoire collective, celle de la communauté imaginaire de la nation, que parle désormais le musée d'histoire ${ }^{31}$. Une exposition d'histoire du travail est la grande attraction de l'exposition de Paris, en 1867 et 1878, qui montre le développement de l'activité humaine à travers 5000 outils et instruments depuis l'âge de pierre jusqu'au début du XIXème siècle. À l'occasion des Expositions universelles de 1878 et de 1900, les deux à Paris, les "Rues des Nations" manifestent les spécificités des vies historiques nationales. Un témoignage spectaculaire de cet intérêt toujours plus grand porté au quotidien domestique est - L'histoire de l'habitation humaine - présentée à l'exposition de 1889 sous la forme d'une série de 23 maisons grandeur nature dessinées par Charles Garnier. Enfin d'autres dispositifs se développent tout au long des foires du XIXème siècle: les restaurants nationaux et les reconstitutions de villages nationaux. En 1900, tel est le paysage offert par l'exposition consacrée au Vieux Paris ${ }^{32}$. Les aspects de petites patries ou de traditions religieuses peuvent trouver leur place au sein des expositions et déboucher sur des musées spécifiques, ou au moins des départements particuliers d'établissements. Une première exposition consacrée à l'art juif en 1878, à partir de la collection Strauss, met ainsi l'accent sur les aspects ethnologiques de cette communauté.

Un basculement des références temporelles

L'exposition d'images ou d'objets présentés comme autant de symboles de l'abjection, destinés à susciter l'horreur, ou au moins à appeler la désapprobation sur un phénomène ou une entité, est un ressort permanent des musées d'histoire contemporains: en ce sens, tout musée d'histoire est un musée de la criminalité historique. La Révolution avait eu peur de la contagion sensualiste des images de la royauté et de la religion, et le musée d'histoire de Lenoir avait mobilisé les monuments de la monarchie afin de montrer la barbarie des temps écoulés. Cette histoire de la criminalité de l'Ancien régime devient au XXème siècle celle de la barbarie des ennemis de la patrie.

La première institution qui documente et expose l'histoire contemporaine du XXème siècle est en effet la bibliothèque et musée de la guerre, fondée en 1917 à l'initiative d'un riche industriel parisien, Henri Leblanc, et de sa femme dans leur appartement de l'avenue Malakoff. Abrité au château de Vincennes à partir de 1925, il est aujourd'hui installé pour la bibliothèque à Nanterre (BDIC) et pour le musée aux Invalides, avec très peu d'espace d'exposition, ce qui le cantonne à des expositions temporaires. L'appel à l'émotion du souvenir, à la
31.Voir ainsi les études réunies par Kaplan (1996).Et,dans une perspective anthropologique, M. Herzfeld (1997)

32. Cf. P. Centlivres, D. Fabre et F. Zonabend (1999) 
damnatio memoriae, est évident dans le Premier catalogue raisonné rédigé en 1916 par Henri Leblanc, quant il écrit: "Durant des siècles, on pourra venir chez nous se remémorer les gloires et les horreurs de cet immense conflit, on pourra se documenter, et du même coup remplir son âme de l'amour de la France et de I'horreur de l'Allemagne et de l'Allemand" 33 .

Le propos est identique au musée de sculpture comparée: Camille Enlart signale au musée, par des étiquettes spécifiques, ceux des moulages dont les originaux ont été endommagés par les Allemands. II ajoute que, passée la stigmatisation des pratiques de la première guerre mondiale,

les étiquettes vert clair se multiplieront quand même au Trocadéro. II conviendra d'en mettre aux monuments de la Gaule romaine ruinés depuis la première incursion des Barbares, aux vestiges de Thérouanne anéantis par leurs descendants en 1553; aux bustes détruits à Strasbourg en 1870. Le musée qui résume l'histoire de notre culture artistique doit signaler désormais à la haine et au mépris des civilisés l'oeuvre qu'ont poursuivie à travers les siècles les ennemis de la civilisation ${ }^{34}$.

Ces épisodes confirment le processus de construction de l'identité nationale dans le sentiment de la perte d'un héritage commun, et vérifient l'intuition de Renan dans la célèbre conférence de la Sorbonne de 1882 intitulée Qu'est-ce qu'une nation?: "En fait de souvenirs nationaux, les deuils valent mieux que les triomphes: car ils imposent des devoirs, ils commandent l'effort en commun" ${ }^{35}$. La commémoration des destructions de la première guerre mondiale fait de ces collections un véritable "lieu de mémoire" national.

Les musées de l'histoire immobile

Les musées des petites patries disparaissent peu à peu après la seconde guerre mondiale: I'ancienne thématique provinciale s'efface avec la mort de leurs identités traditionnelles. Toute l'oeuvre historienne d'un Philippe Ariès en porte témoignage, avec la nouvelle inflexion d'une histoire des mentalités qui se nourrit de l'inspiration traditionaliste déçue ${ }^{36}$. "L'histoire immobile" chère à Emmanuel Le Roy Ladurie triomphe pour peindre le "monde que nous avons perdu" (Peter Laslett) au sein de musées d'art et tradition populaire dont le réseau, initié par GeorgesHenri Rivière, éclipse des musées d'histoire définitivement vieillis. L'idéal du musée laboratoire au service de l'ethnographie française s'incarne par exemple au musée de Beaune au cours des années 1950-1960³. Au cours de la décennie 1970-1980 c'est dans certains musées de la seconde "génération Rivière" que s'expose la modernité de l'école des Annales, et que s'opère la mise en scène d'une histoire sociale "vue d'en bas". Ainsi, le musée de Bretagne à Rennes se veut une forme d'auto-représentation d'une communauté: I'exposition de "sacs à procès" illustre la justice de l'âge classique, selon des modalités qui reprennent à leur compte les lectures de Pierre Goubert et de l'école des secondes Annales. L'effort est moins accompli pour l'histoire immédiate, dont des dispositifs 
médiatiques - télévision, journaux - sont censés rendre compte de manière un peu paresseuse.

L'invention de l'écomusée ou du musée de société a largement participé d'un mouvement de retour sur soi de l'anthropologie, du lointain au proche: I'anthropologie de la France s'est élaborée à ce moment, remplaçant en quelque sorte l'anthropologie de l'autre par celle du même ${ }^{38}$. Les causes en sont complexes, qui mêlent le processus de décolonisation, la reconversion de l'anthropologie universitaire, enfin l'élaboration d'une demande publique grâce à la Mission du Patrimoine ethnologique, qui est venue complexifier la situation entre la recherche et les musées ${ }^{39}$.

Une génération de musées de mémoire

Les années 1960 voient le 20ème anniversaire de la Libération, et la fondation de musées de la Seconde Guerre mondiale, à l'initiative d'associations ou d'amicales. Ces musées sont directement porteurs d'une mémoire et d'idéaux; ils sont souvent situés sur un lieu symbolique - camp du Struthof, lieu d'exécution de la citadelle de Besançon, ou territoire de maquis et de combats comme le Vercors. Le musée de la résistance nationale de Champigny-sur-Marne date de 1965; installé dans un hôtel particulier du XIXème siècle, en bordure de la Marne, dans un parc baptisé du nom de Vercors, pseudonyme du fondateur des Editions de Minuit clandestines, il rassemble les plus importantes collections relatives à la résistance française pendant la Seconde Guerre mondiale, fruit de plus de 2000 donations et de dépôts privés ou publics. Plus largement, il veut témoigner de I'histoire sociale française de 1929 à 1947.

La présentation de l'actuel musée se fait toujours l'écho de sa fondation:

Dans les années 1960, la nécessité de bâtir une structure afin d'enseigner la Résistance et de pérenniser la mémoire de cette époque exemplaire se fit jour. En effet, en ces temps encore très proches de la fin de la guerre, la politique de mémoire initiée par les pouvoirs publics restait traditionnelle dans l'esprit et dans la forme D'anciens Résistants lancèrent alors l'idée d'un Musée de la Résistance nationale. Ils se constituèrent en association et, pendant 20 ans, collectèrent auprès de leurs camarades de lutte et de leurs familles documents et objets ${ }^{40}$.

A Grenoble, c'est en 1963 que des Anciens combattants de la Résistance, des enseignants, réalisent avec l'aide des Archives départementales de l'Isère, une exposition sur la Résistance dauphinoise. Son succès conduit à un Musée permanent inauguré en 1966, dans un local de la ville de Grenoble ll'appartement natal de Stendhal), qui devient en 1970 le Musée de la Résistance et de la Déportation soutenu par Pierre Mendès-France, alors député de l'Isère.

Vingt ou trente ans plus tard, les derniers témoins disparaissent, et les associations issues de la Résistance et de la déportation en viennent à s'effacer peu à peu. L'urgence est alors de repenser les formes de la transmission d'engagements et de militantisme, en particulier afin d'assurer le devenir du patrimoine de ces musées, de leurs archives et de leurs collections. Il faut enfin et
38. Cf. Florence Weber (2003).

39. Deux ouvrages à caractère partiellement autobiographique sont à cet égard utiles: Martine Segalen (2005) et Jean Cuisenier (2006)

40. Museé de la Résistance de Grenoble, d'après le Site Web http://www.resistanceen-isere.com. 
41. Cf. Claude Quetel (1992). Voir l'analyse critique de Benjamin C. Brower (1999).

42. Cf. Caroline Barcellini (2003). surtout réfléchir aux nouvelles fonctions de ces musées à l'égard de visiteurs dépourvus de la mémoire d'une génération. La génération des anciens combattants ou des maquisards, fondateurs des premiers musées, s'efface et doit envisager d'autres modalités d'appropriation et de médiation des collections qu'elle a réunies. Lors d'une enquête de l'IHTP, voici quelques années, à propos de la commémoration, Mona Ozouf avait fait remarquer que dans bien des cas les musées locaux consacrés à la seconde guerre étaient des musées où se réaffirmait le sentiment d'avoir vécu ensemble - quelle que soit l'insignifiance des événements précis qui y avaient été vécus. II ne s'agissait donc pas de participer de la grande histoire, mais d'affirmer une mémoire à soi, de rappeler avoir été ensemble - et le cas échéant de manière solidaire. La gamme des musées d'histoire peut ainsi comprendre des variations tout à fait considérables, depuis l'événement traumatique - la destruction d'Oradour - jusqu'au rappel des privations de nourriture - le musée de la vie quotidienne sous l'Occupation, qui réunit semelles de bois articulé et tickets de rationnement.

Caen et Péronne constituent deux symboles, à certains égard opposés - l'un est un musée d'idées et de l'implication, l'autre est un musée d'objets - de la nouvelle génération des musées de guerres. Le mémorial-musée de la Paix est inauguré à Caen en 198841. L'historial de la grande guerre ouvre à Péronne en 1992. Le musée de Péronne est le seul, de tous les musées actuels, dont on peut dire qu'il témoigne directement d'un travail historien, mené au sein du Centre de recherches qui a été adjoint au musée proprement dit. Mais les deux participent de lieux de mémoire conscients d'eux-mêmes comme l'avait annoncé Pierre Nora - les Lieux de mémoire datent de 1984 pour le ler volume. II s'agit aussi d'une rupture avec le musée d'histoire typique qu'est le musée militaire: à savoir le musée de l'Armée, créé en 1905 par la fusion du musée d'Artillerie (né sous la Révolution et installé en 1871 aux Invalides) et du musée historique de l'Armée, fondé en 1896 par une société, La Sabretache, dont le président, le peintre Edouard Detaille, souhaitait constituer, à partir de ses propres collections, un musée militaire national sur le modèle des salles rétrospectives de l'Exposition universelle de Paris de $1889^{42}$.

A Caen il s'agissait de créer "un nouveau type de musée" grâce à un "voyage dans l'histoire", à une mise en scène revendiquée. La visite se termine par 3 films, dont le D Day est le plus populaire. Mais les visites ne décollent pas en regard de la fréquentation in situ des musées privés ou locaux installés sur les plages. Pour diverses raisons, le mémorial de Caen a, au cours des années 1980-1990, cristallisé les polémiques entre historiens et intellectuels critiques. De J.P. Rioux et des historiens de I'IHTP aux philosophes du Collège de philosophie d'abord associés au projet (pour J.-L. Déotte au moins), jusqu'aux critiques d'historiens étrangers (D. J. Sherman), le mémorial a été l'objet privilégié d'analyses parfaitement contradictoires - exactement comme l'écomusée du Creusot sur un autre plan, faisant ainsi de l'établissement un tournant dans la représentation muséale de l'histoire en France. Le legs muséographique, en particulier, est évident: le futur Mémorial Charles de Gaulle prévu à Colombey-les-Deux-Eglises en 2008 est installé dans un bâtiment réalisé par les architectes Millet-Chilou, concepteurs 
du mémorial de Caen, installé au pied de la colline où se dresse aujourd'hui la Croix de Lorraine et de vastes dimensions.

Le musée de Péronne est également dirigé contre la tradition figée des musées d'histoire: le terme choisi d'Historial combine Histoire et mémoire. Stéphane Audouin-Rouzeau, historien étroitement associé à l'institution, écrit ainsi en 1992 que l'historial est aussi mémorial, dans un article d'Historia intitulé La grande guerre prend fin aujourd'hui ${ }^{43}$. En 1987 I'Historial organisa un mouvement appelé Faites entrer votre nom au musée, pour collecter les objets: d'une part les noms des donateurs seront consignés dans les activités de l'Historial, d'autre part les objets recueillis seront "une part inaliénable du patrimoine historique national" (Le Courrier picard, 1987). Ceci est à mettre en rapport avec diverses initiatives très médiatiques à propos de "parole des poilus" (France Inter) comme avec la valeur esthétique et pécuniaire croissante des objets de tranchées, au fur et à mesure qu'une littérature érudite se déploie sur le sujet.

On pourra reprendre le diagnostic porté par Sophie Wahnich:

I'historial de Péronne est un lieu pour écrire une histoire qui juge et qui condamne la conflit et qui travaille à son effacement. A ce titre l'historial est le lieu de l'invention d'une mémoire européenne pour le présent [...] Caen laisse peu de place à la mémoire de la seconde guerre mondiale. II en propose surtout une histoire très construite [...] Pour le temps présent, il juxtapose à cete histoire des discours idéologiques qui ne sont pas à proprement parler une élaboration de la mémoire de la guerre, et des mémoriaux qui à l'échelle du débarquement et de la bataille de Normandie ne sont que des évocations de ce qu'il faudra bien aller chercher ailleurs ${ }^{44}$.

Au-delà de ces deux grans établissements, les musées d'histoire locale ont évidemment été présents dans les rénovations et les constructions de musées menées à bien entre la fin des années 1970 et la fin de la décennie 1990 et qui s'élèvent à environ 300. Ces trente dernières années ont vu une évolution décisive des musées d'histoire, dans les politiques culturelles territoriales surtout, au fil des volontés politiques locales, ainsi qu'une mutation des professionnels et de leurs pratiques. Telle est, par exemple, la création en 1984 d'un musée sur la commune d'Estivareilles (musée départemental de l'Armée Secrète et de la Résistance) ouvert en présence de Lucien Neuwirth, grande personnalité de la Résistance et de l'Armée Secrète de la Loire.

Les musées d'histoire sont entrés dans les politiques culturelles ainsi que dans les actions menées en faveur du développement touristique - avec les écomusées, les musées de société, les musées de site archéologique, les maisons de parc régional etc. ${ }^{45}$ L'une des singularités du musée d'histoire est qu'il engage souvent un partenariat entre l'Etat et les collectivités locales, contrairement aux grands musées nationaux qui relèvent seulement de l'administration centrale. En ce sens, son histoire institutionnelle reflète à bien des égards les spécificités de cette relation. D'une part le modèle des musées nationaux, tel qu'il a été configuré par la Direction des Musées de France, est très important du point de vue administratif et symbolique, quant aux définitions de l'institution musée, quant à ses orientations et à ses activités ${ }^{46}$. D'autre part, les collectivités se sont volontiers engagées dans
43. Cf. Audouin-Rouzeau (1992, p. 94).

44. Cf. Wahnich (2005, p. 8081).

45. Cf. J.-C. Duclos (1992); voir aussi Philippe Ifri, Maurice Gueneau et Philippe Maubant (1997).

46. Le musée d'art constitue le coeur de cette définition des établissements, à quelques exceptions près. La reconnaissance tardive des musées d'histoire relève de la négligence plus largement portée aux autres types de musée que le musée d'art. 
47.Ainsi, davantage qu'un musée d'histoire, le Musée de Bretagne, créé en 1975, se définit comme un musée de synthèse. Conçu comme un musée de société, il mêle étroitement les sciences humaines telles l'archéologie, l'ethnologie et l'histoire. N'excluant nullement les questions contemporaines, $\mathrm{i}$ restitue également l'histoire de la Bretagne et celle de son identité. Cf. Elsa Chevalier (2001). L'écomusée d'Alsace, ouvert en 1984 sur une vingtaine d'hectares entre Strasbourg et Mulhouse, est à la fois un musée, qui possède des collections d'objets et un parc historique, offrant des animations historiques et fokloriques autour de thèmes festifs, carnavalesques ou agricoles. Un train touristique relie le village à la mine de potasse Rodolphe. L'ensemble accueille bon an mal an 300000 visiteurs, mais rencontre des difficultés de gestion et de légitimité.

48. Cf. Emmanuelle Francois (1996).

49. Nicolas Georges, rapport de 2001

50. Cf. Serge Barcellini (2005). les musées de société et d'histoire, au fur et à mesure que les enjeux territoriaux et culturels semblaient l'imposer. Elles pouvaient trouver dans des établissements qui substituaient la perspective patrimoniale régionale à une perspective exclusivement disciplinaire un élément satisfaisant de leur politique de culture et de communication ${ }^{47}$. Mais la quasi-absence des musées dans les lois de décentralisation peu à peu votées, comme dans beaucoup de dispositifs de partenariat contractuel, ou leur faible place dans les dispositifs de la déconcentration du Ministère de la Culture - les postes de conseillers musées au sein des Directions en régions (les DRAC) ont été pourvus très lentement - ont abouti à un retard par rapport à d'autres engagements et à d'autres chantiers, comme celui du spectacle vivant.

L'investissement des collectivités locales doit se garantir d'exigences scientifiques et muséographiques nouvelles: à l'Inspection de la DMF une jeune chartiste, Marie-Hélène Joly, est en charge des musées d'histoire, à laquelle succède Nicolas Georges. Une association des musées d'histoire voit le jour simultanément. La nécessité de disposer d'un état des lieux des musées d'histoire de la Seconde Guerre mondiale se fait évidente, afin d'esquisser le fonctionnement des musées, leurs statuts, la question des propriétés des collections, leurs publics, leur typologie ${ }^{48}$. Comme le dit l'Inspection des musées en 2000,

Ces musées souffrent d'un manque de réflexion sur l'extension même de la notion de résistance. L'avenir de ces musées ne pourra faire l'économie d'une réflexion nationale. Car si la création s'est faite au gré des initiatives locales et associatives, la disparition progressive de maintes petites structures devra être encadrée et faire l'objet d'une réflexion globale ${ }^{49}$.

Les rassemblements de hasard, le plus souvent, d'objets personnels que sont les premiers musées de la Résistance, doivent être réexaminés à la lumière de critères scientifiques, de conservation, comme d'éventuelles exigences de fréquentation touristique. II reste que l'intervention de l'Etat est demeurée faible dans les quelque 200 musées relevant de la catégorie des musées de guerres mondiales ${ }^{50}$.

Le Guide des musées et collections d'histoire en France paru en 1996 sous la direction de Marie-Hélène Joly affirme que

la sauvegarde n'a de sens que si elle est organisée et structurée. La simple présentation au public ne suffit pas - car contrairement aux musées des Beaux-Arts - la délectation esthétique n'est pas le seul objet. Elle est une partie, avec la curiosité, l'étonnement, le souvenir parfois, du propos. L'autre versant reste la signification de l'objet pour l'explication de l'histoire ou de l'institution (UOLY, 1996).

C'était là affirmer les valeurs spécifiques du musée d'histoire contre le musée des beaux arts, modèle hégémonique dans la tradition française de la DMF. Le but du musée d'histoire est de servir le travail d'explication de l'historien, ce qui l'oblige à fournir aux historiens des outils spécifiques - en l'occurrence des clés de lecture des images (matériel de propagande, affiches etc.). Ce que j'appellerai le moment disciplinaire du musée d'histoire est marqué en 1996 par le colloque 
de l'Historial de Péronne coordonné par Marie-Hélène Joly et Thomas Compère Morel, Des musées d'histoire pour quoi, pour qui? édité ensuite sous le titre: Des musées d'histoire pour l'avenir (1998) ${ }^{51}$.

La leçon a porté: Robert Bresse, Directeur du musée de l'Armée, chargé de la maîtrise d'ouvrage de la modernisation du musée de l'Armée qui va s'échelonner sur 5 ans déclare ainsi aujourd'hui: "Ma mission est de transformer un musée d'objets en musée d'histoire" 52 . Telle est l'une des orientations de la rénovation du musée de l'Armée, conduite en cinq tranches sensiblement égales d'une quinzaine de millions d'euros chacune: le creusement total d'une des cours des Invalides pour installer l'historial Charles de Gaulle est un chantier spectaculaire, pour un dispositif essentiellement audio-visuel. L'Historial devrait constituer un "monument audiovisuel" structuré autour du son et de l'image, "matériaux muséographiques gaulliens" par excellence, sans la participation d'aucun objet ${ }^{53}$. Comme le déroulement d'un film, le déroulement d'une visite au sein de ces mémoriaux ne permet pas le retour en arrière, la comparaison, l'arrêt sur l'image pour l'expliquer: c'est un appel à l'émotion, à la réaction, sans donner place à l'engagement et à la réflexion. Une esthétique du choc, comme le suggère le philosophe J.-L. Déotte, gouverne alors l'expérience du musée. Plus largement, le spectacle du musée s'intègre alors dans un rapport gouverné par l'émotion.

Les nouveaux musées de l'éthique et du développement durable - ou du divertissement bien-pensant

La thématique de la mémoire juste (P. Ricoeur) ou plus largement la révision critique des lieux de mémoire nationale fait du musée d'histoire un nouveau lieu de reconquête civique, spécifiquement républicain à partir des années 1990, contre la montée de l'extrême droite notamment et d'éventuels dérapages antisémites. L'agenda politique national s'empare à nouveaux frais de ces établissements, en collaboration ou en concurrence avec certaines associations ou avec certaines collectivités locales. En considérant la nouvelle configuration des musées consacrés à la seconde guerre mondiale, Jean-Yves Boursier juge ainsi que "Nous sommes passés de l'altérité (le musée supporté par un groupe) à la norme et au discours moral, appuyés par des opérations de communication" ${ }^{154}$. De là l'apparition de lieux qui ne relèvent pas du genre du musée d'histoire, mais bien du genre singulier d'un "mémorial hors sol" selon la curieuse formule de Gilles Vergnon, car il porte un message suivant: "I'horreur de la guerre, le dégoût de la barbarie, la compassion pour ses victimes, mais aussi la déshistoricisation de la Résistance, immergée dans l'éternel combat du Bien contre le Mal"55.

Le trend funèbre du musée d'histoire en France est-il à rapprocher du dark tourism ainsi baptisé par John Lennon, un spécialiste britannique de l'économie du tourisme? ${ }^{56}$ A la fois exorcisme d'un trauma, instrument de devil, le mémorial de Caen a choisi d'élargir la transmission de la Première Guerre mondiale tant chronologiquement que géographiquement. De là, l'ouverture sur la guerre froide
51 Organisé en novembre 1996 à Péronne, à l'initiative de l'Historial de la Grande Guerre et avec le concours du Ministère de la Culture et du ministère des Anciens combattants, le colloque - Des musées d'histoire pour qui? pour quoi? - aborde ainsi cinq grands thèmes tout à fait représentatifs des mutations alors en cours, afin de considérer les musées d'histoire dans le contexte général de diffusion du savoir historique. Tant la conquête des publics que la médiation et plus particulièrement l'utilisation des nouvelles technologies, que l'enjeu social ou les enjeux territoriaux étaient ainsi examinés.

52. Réponse aux questions des intervenants du site L'Internaute, consulté le 31 janvier.Voir aussi REGARDS sur l'actualité du musée de l'Armée, 2007. Un protocole Défense-Culture signé le 17 septembre 2005 organise les perspectives en matière patrimoniale et muséographique des forces armées.

53. Une salle multi-écrans de 200 places en constituera le coeur entouré d'un anneau de déambulation et d'exposition, ouvrant sur de petites salles conçues comme des laboratoires techniques, pour voir les textes et les images projetées.

54.Cf.Boursier (2005, p.241).

55. Cf. Gilles Vergnon (2005, p. 162).

56. Voir J. J. Lennon et M. Foley (2000). 
57. Situé à $5 \mathrm{~km}$ de SaintOmer, installé dans un ancien ouvrage allemand, le Centre d'histoire de la Coupole a été inauguré en 1997 autour de films d'archives rares, de photographies géantes, et de spectacles multimédias. L'originalité du centre tient à la présentation des rapports entre l'innovation technique, les sciences et la guerre, en quatre langues (français, anglais, néerlandais, allemand). Le Simserhof, l'un des ouvrages les plus importants et les mieux conservés de la ligne Maginot, confié au département de la Moselle et à la communauté de communes de Bitche, a fait quant à lui l'objet d'un programme de mise en valeur culturelle et touristique inauguré en 2002.

58. On trouvera une comparaison possible avec l'ImperialWar Museum et le musée d'Ypres dans Andrew Whitmarsh (2001).

59. Cf. Young (1993b). et les mouvements pour la paix dans le monde actuel. Péronne a choisi la perspective européenne, voire internationale, de même que la Coupole, centre d'histoire et de mémoire du Nord-Pas de Calais, centré sur l'histoire militaire et sur l'histoire des techniques en relation avec des musées britanniques, allemands et belges ${ }^{57}$. Le Centre européen du résistant-déporté dans le camp du Struthof veut représenter les exemples les plus différents de l'engagement contre le nazisme. Ce musée, entièrement repensé, est désormais consacré à l'histoire du camp de concentration de Natzweiler et de ses camps annexes, à partir des anciennes collections, ainsi que des objets des principaux camps de concentration et d'extermination, puisqu'il s'agit de fournir des informations sur les 14 principaux camps de concentration installés en Europe. Non loin de là, le Mémorial de I'Alsace Moselle, à Schirmeck, dans un vaste bâtiment à la façade de verre, consacre une scénographie exceptionnelle à l'histoire d'une frontière fluctuante qui, de 1870 aux lendemains de la Seconde Guerre mondiale, pèse encore sur l'identité de toute une région et présente également les bases de la construction européenne. Ailleurs, au musée de la résistance et de la déportation de Grenoble, on veut évoquer la thématique des crimes contre l'humanité et les génocides du Rwanda et du Cambodge.

Le désenclavement de la muséologie historique française passe par l'affiliation à des réseaux internationaux d'établissements, par exemple au sein de l'ICOM ou d'associations spécialisées. En ce sens, les nouvelles constructions ou les rénovations témoignent d'exigences professionnelles de qualité internationale mais encore de certaines modes muséographiques liées au tourisme international, du succès de certains architectes dont le marché est lui-même global, enfin des effets de légitimation recherchés par la quête de modèles unanimement reconnus ${ }^{58}$. L'exemple le plus significatif, peut-être, est celui du programme de la Cité de I'immigration au MAAO. Jacques Toubon, ancien ministre de la Culture et patron du projet, a fait le tour du monde des musées d'immigration en quête d'inspiration avec son équipe. Un colloque international sur les musées d'immigration dans le monde a été organisé à la BNF, enfin l'aveu d'une inspiration d'Elllis Island a été maintes fois réitéré par le ministre et par son équipe.

Dans un pays qui n'avait pas bâti, sauf exceptions, de musées d'histoire jusque-là, les dernières décennies ont vu fleurir les constructions de musées, ce qui est en soi une nouveauté. Ces édifices se veulent partie prenante de la stratégie d'interprétation du musée. C'est ainsi qu'à Caen et à Péronne l'image de la fracture a été privilégiée, signifiant le statut de ville martyre de la Deuxième Guerre mondiale pour l'une, et pour l'autre les meurtrissures des champs de bataille de la Somme. Henri Ciriani a choisi à Péronne la faille pour symboliser la tranchée et la rupture de la guerre, et a joué sur le vocabulaire post-corbuséen. Le syndrome de la "tombe absente" évoquée par James Young à propos de l'architecture enterrée des mémoriaux et autres musées ${ }^{59}$. Ailleurs encore, à Oradour ou en Vendée, il s'agit de bâtiments qualifiés par un journaliste du Monde de "furtifs", c'est à dire qu'ils disparaissent dans le paysage, s'effaçant derrière la mise en valeur d'un site de mémoire ou derrière un environnement naturel dont il s'agit de rendre compte dans une perspective de développement durable. 
L'Historial de la Vendée, géré par le conseil général, et situé près de La Roche-sur-Yon, au village de Lucs-sur-Boulogne, a coûté 14 millions d'euros pour le musée seul, auxquels s'ajoutent quatre millions pour la scénographie. Soit, d'après son conservateur, 60 "deux fois moins cher que la moyenne des musées de France". La particularité architecturale est d'être recouvert par une toiture végétalisée de $8000 \mathrm{~m}^{2}$, imaginé par Plan 01, un collectif de quatre cabinets d'architectes parisiens. L'intérieur du bâtiment, de caractère industriel, est modulable, à partir d'un hall de $1020 \mathrm{~m}^{2}$ qui gouverne sept espaces correspondant à des périodes historiques, en partant de la Préhistoire. Sur le modèle du multiplex cinématographique qui a connu ces dernières décennies un grand développement à travers la France, tout est conçu pour rendre le musée ludique, avec une cafétéria, une boutique, un musée des enfants unique en France. Les expositions, toutes temporaires, illustrent des Vendéens célèbres, comme Richelieu, et mettent en scène la guerre de Vendée. Une telle réalisation rompt complètement avec l'installation précédente d'un écomusée de la Vendée, comme avec la création d'un célèbre spectacle historique sous la forme d'un cas relativement unique de Re-Enactment dans la société française contemporaine, le Puy-du-fou.

II y a quelques années deux spécialistes des sciences sociales posaient cette question:

L'utopie puyfolaise va-t-elle continuer à se déployer en mobilisant toujours ou bien va-t-elle s'effriter dans la gestion, dans la concurrence ou dans la conformité avec des normes culturelles capables d'attirer un public d'autant plus large qu'elles seront moins rivées à la célébration d'une société aujourd'hui disparue $?^{61}$

On constate aujourd'hui que le musée d'histoire de la Vendée annonce avec d'autres sans doute ailleurs en France - un ensemble de reconfigurations institutionnelles et historiennes ${ }^{62}$. II incarne un équipement conforme aux normes du loisir contemporain, capable de concilier éducation et distraction de loisir, aux dépens de formes traditionnelles d'érudition locale et peut-être du maintien d'une qualité muséographique nationale telle que l'Inspection des musées a toujours tenté jusque-là de la maintenir. En cela il reflète les nouvelles conditions de financement et de réalisation de ces équipements, comme le nouveau statuł d'une histoire qui cultive autant le marché touristique que la démarche identitaire - la construction de l'identité vendéenne étant dans l'histoire savante un topos classiquement réinterrogé, qui n'apparaît pas ici déterminant.

L'histoire de l'immigration constitue un enjeu symétrique, si l'on ose dire, du cas vendéen. Les expositions du musée dauphinois consacrées aux minorités présentes dans l'agglomération grenobloise - les Grecs, les Italiens, les Maghrébins...- sont la première manifestation de quelque ampleur d'une reconnaissance des communautés et de l'histoire de l'immigration dans les musées français ${ }^{63}$. D'après leur conservateur, J.-C. Duclos, "le musée de patrimoine régional ne peut prétendre remplir pleinement ses missions s'il limite son domaine $d^{\prime}$ intervention aux seules périodes historiques et aux seules cultures d'origines" 64 .
60. Conservateur M.Vital; cf. Allix (2006).

61. Cf. Jean-Clément Martin et Charles Suaud (1996, p. 177).

62. Citons quelques établissements d'un nouveau genre, le Compa à Chartres, Ruralies près de Niort, pour prendre des exemples volontairement hétéroclites de muséographie "historique".

63. DUCLOS (1999)

64. Cf. Duclos, ibidem p. 8. 

(2002).

67. Cf. Claude Lévi-Strauss (1962, p. 346-347).
Ainsi, le musée intègre dans sa démarche l'histoire contemporaine, voire immédiate, et de plus en plus les cultures étrangères comme objet d'exposition. Dans ce dessein, l'institution a conçu et réalisé depuis 1989 une série d'expositions évoquant la mémoire des communautés qui composent la population iséroise avec par exemple: L'Italie des Pouilles (Corato: Grenoble en 1989), la Grèce (Des Grecs en 1993), I'Arménie (D'Isère et d'Arménie en 1997) et la communauté maghrébine en 2000 (D'Isère et du Maghreb, Pour que la vie continue...). Ce cycle d'expositions consacrées à l'investigation scientifique sur les identités transplantées dans le Dauphiné a pour objectif de "constituer la mémoire collective, de contribuer à l'apprentissage de la différence, du respect des cultures et du partage d'une même identité, futelle composite" 65 . En fait, les études de publics menées à ce propos concluent plutôt que le musée enseigne la reconnaissance de ressemblances. La muséographie employée - à savoir la présentation de personnages qui apparaissent comme autant de porte-parole de leurs différentes communautés - évoque le mode nord-américain de faire appel à d'authentiques histoires de vies ou à des témoignages scénarisés par une équipe - voire à des fictions pures et simples - pour communiquer aux visiteurs une vue "participative" 60 . La muséologie de l'immersion, terme couramment employé aujourd'hui à propos des musées des sciences, a largement touché le musée d'histoire. Le musée français use plus modestement et plus timidement qu'aux Etats-Unis et qu'au Canada de l'histoire-fiction pour ses scénarios, mais la tendance est néanmoins très repérable - dans la première version de l'exposition du musée du maquis du Vercors, à propos de l'assassinat d'une petite fille. En particulier, tel semble l'enjeu de l'oralité au musée, de la présence de récits et de bruits - dans le musée de la résistance de Grenoble en particulier, en accompagnement d'un streetscape.

Une histoire-mémoire à différentes échelles

Par là, le musée d'histoire illustre particulièrement le dilemme entre "une histoire qui apprend plus et explique moins, et une histoire qui explique plus et apprend moins" que soulignait Claude Lévi-Strauss voici une génération:

[Car] l'histoire biographique et anecdotique, qui est tout en bas de l'échelle, est une histoire faible, qui ne contient pas en elle-même sa propre intelligibilité, laquelle lui vient seulement quand on la transporte en bloc au sein d'une histoire plus forte qu'elle; et celle-ci entretient le même rapport avec une classe de rang plus élevé. Pourtant, on aurait tort de croire que ces emboîtements reconstituent progressivement une histoire totale; car ce qu'on gagne d'un côté, on le perd de l'autre. l'histoire biographique et anecdotique est la moins explicative; mais elle est la plus riche du point de vue de l'information, puisqu'elle considère les individus dans leur particularité, et qu'elle détaille, pour chacun d'eux, les nuances du caractère, les détours de leurs motifs, les phases de leurs délibérations. Cette information se schématise, puis s'efface, puis s'abolit, quand on passe à des histoires de plus en plus "fortes" ${ }^{167}$. 
Pour sortir par en bas, en somme le musée d'histoire devient le musée biographique de tel ou tel personnage; et pour sortir par en haut, il devient ou redevient le musée de l'homme. Aujourd'hui l'un des enjeux du nouveau musée de Marseille est de fait une historicisation du musée des ATP. Georges-Henri Rivière n'était pas historien, et le musée présentait une vue éternelle des traditions françaises dans un rapport d'empathie, de connivence, aux objets de la tradition. Au contraire, d'après son nouveau directeur, Michel Colardelle, le futur établissement doit considérer les objets comme profondément inscrits dans I'histoire. C'est contre l'idéologie Malraux, comme il l'appelle, que se joue l'appel à la contextualisation - celle de la Méditerranée contemporaine, dans un questionnement qui se décline en 5 thèmes, renouvelés tous les 5 ans: le Paradis, I'eau, le chemin, la cité, le Masculin/Féminin.

L'appel à la mémoire est devenu un leitmotiv de l'histoire "au second degré"68. Entre la formule de Paul Valéry à propos de la nouvelle conscience de la mortalité des civilisations et celle de Daniel Halévy à propos de l'accélération inédite de I'histoire se fait jour un besoin de se rassurer quant à la permanence de la nation, de la société ou de la communauté - telle est au moins la thèse d'une compensation de l'accélération de l'histoire pointée par Hermann Lübbe et développée par lui à propos de la muséalisation contemporaine en général.

Par rapport à la situation des années 1950-1970, les musées d'histoire bénéficient aujourd'hui d'une image renouvelée et d'un préjugé plus favorable dans le public ${ }^{69}$. Is ont tenu compte des modifications des ambitions et des pratiques des historiens: suivant en quelque sorte le constat de Pierre Nora quant à l'éclatement de l'histoire, les expositions en leur sein sont devenues très thématisées et spécialisées, voire fragmentées, tout en conservant leur revendication d'autorité et leur anonymat d'auteur, ce qui ne va pas sans poser problème à I'heure de l'ego-histoire. L'actualité présente est marquée par la multiplication des musées-mémoriaux, qui vont de l'histoire coloniale de l'Afrique du Nord aux mémoriaux de sites, aux mémoriaux de victimes et aux musées de la paix. C'est ce que Annette Becker nomme les "musées ouverts", créés sur les lieux mêmes des drames qu'ils commémorent ${ }^{70}$. Les intentions sont largement marquées par une éthique des droits de l'homme, et s'inscrivent dans le présent de manière beaucoup plus explicite que dans les musées d'histoire traditionnellement conçus comme consacrés à l'enseignement de l'histoire. Une sorte de devoir d'implication des visiteurs est ici à l'agenda des musées d'histoire.

Le musée d'histoire jouit d'une très grande crédibilité parce qu'il expose l'authenticité par excellence, en présentant des témoins véridiques du passé, présentés comme autant d'évidences d'une présence du passé, et qui valent pour preuve de la véracité des propositions du musée. Mais le musée d'histoire, aujourd'hui, est bien davantage un musée du présent qu'un musée d'histoire au sens traditionnel du terme. Dans les musées des années 1980, "I'immersion dans les pratiques du passé, loin d'être nostalgique, éveille aux problèmes du présent" ${ }^{\prime \prime 1}$. L'écomusée, en particulier, élabore une nouvelle représentation du patrimoine conçue comme prise de conscience d'elle-même par la société, grâce à la mise au jour (interminable) de ses "propriétés"72. II participe en ce sens d'une dynamique
68. Cf. Jean-Pierre Bacot et Christian Coq (1999).

69. Cf. Olivier Donnat,(1994).

70. Voir Annette Becker (1998).

71. Préface de la brochure de la Fédération des Ecomusées, 1990. Voir aussi Isabelle Lazier et André Desvallées (1987).

72. Voir le manifeste de Terrain, la revue de la Mission du Patrimoine ethnologique du Ministère de la Culture (FLEURY, 1988). Un bilan de cette ethnologie est fourni par M.Segalen (1989) et Benzaïd (1980). 
73. Cf. F. Raphaël et G. Herberich-Marx (1987).

74. Cf. D. Löwenthal (1985).

75. Voir les communications réunies dans J.-Y. Boursier (1997). Signalons parmi une très abondante bibliographie deux rares thèses comparatives sur le thème:Anna Balzarro (2001) et Isabelle Benoît (2001). On n'évoque pas ici le cas spécifique des musées de l'holocauste; voir J. E. Young (1993).

76. Cf. Daniel J. Sherman (1995).

77. Cf. Daniel Fabre (2000). inédite du "patrimoine" dans la société, dont témoignent aussi les recherches commanditées par la Mission du Patrimoine sur les pratiques et les politiques culturelles de l'identité. Si le musée d'histoire classique mobilisait le passé pour l'avenir, l'écomusée, lui, figure plutôt, selon une excellente formule de Freddy Raphaël, "une provocation de la mémoire"73. L'utopie des écomusées Rivière des années 1970 a tenté de donner une position d'acteurs aux visiteurs - même si, comme l'a montré Octave Debary à propos du Creusot, c'était sur l'échec d'un travail de mémoire inachevé que de telles thèses reposaient parfois.

Au siècle dernier, la patrimonialisation s'attachait à rendre une voix aux monuments désormais silencieux, à tous les restes du passé, dans un sentiment d'urgence dû à la hantise de la perte. Le champ patrimonial était "un autre pays", plus beau ("Time beautifier of things") d'être étranger et disparu ${ }^{74}$. Le musée d'histoire contemporain veut plutôt conférer une signification au vécu des jours, à travers la série des expositions, la succession des points de vue et des cadrages d'une population et d'un territoire. En ses tâtonnements, voire ses errements, cette nouvelle figure de musée manifeste la recherche d'une alternative à la construction du siècle dernier. Bien des musées d'histoire ou d'archéologie, qui connaissent un développement spectaculaire, satisfont à une préoccupation patrimoniale, mais se veulent directement liés aux mouvements sociaux ou communautaires, ou encore à l'intérêt que suscitent les nouveaux thèmes de l'après-modernité : I'identité, l'éthique, le genre sexuel...Le cas des musées de la Résistance est aussi particulièrement révélateur d'enjeux présents ${ }^{75}$.

$\mathrm{Si}$, au terme d'une histoire complexe, le musée d'histoire fait figure d'institution centrale et de nos jours peu contestée de la culture occidentale, il recouvre désormais des réalités si diverses, quant aux collections qu'il mobilise et aux démarches dont il se réclame, que sa définition en apparaît durablement menacée. Surtout, la légitimité de la conservation et de la mise en valeur du patrimoine historique repose aujourd'hui moins sur le respect de l'intention des ancêtres qu'il donnerait à comprendre et transmettre que sur l'intérêt général du public - et de communautés - pour la mémoire, son travail et ses représentations. Reprenant le théoricien critique post-moderne Andreas Huyssen, Daniel J. Sherman souligne ainsi, à l'issue de sa comparaison des musées de Péronne, Verdun et du mémorial de Caen, que l'intérêt des musées d'objets est de demeurer ouverts aux multiples discours de la mémoire - contre, à la fois, une marchandisation spectaculaire de I'histoire qui exclut l'approche critique et savante, et une fusion du propos muséographique et du savoir historien qui suscite en retour une frustration de la mémoire ${ }^{76}$. L'intérêt contemporain porté aux hauts lieux, aux monuments historiques, aux châteaux privés et autres demeures ou territoires touchés par la conversion patrimoniale est d'ailleurs devenu en quelques années un sujet d'interrogation et d'étude pour l'ethnologue. C'estl'habitant et le visiteur qui concentrent ici l'attention, dans une relation qui entend "domestiquer l'histoire"77. Le rapport du patrimoine et de l'écriture de l'histoire devient un enjeu d'actualité pour les musées d'histoire, dans une relecture des constructions de l'identité et des traditions, entre continuité d'intentions et déplacement des horizons de référence. 


\section{RÉFÉRENCES}

ADHÉMAR, Jean. L'éducation visuelle des fils de France et l'origine du Musée de Versailles. $L a$ Revue des arts, Paris, n. 6, p. 29-34, 1956.

. L'enseignement par l'image. Gazette des Beaux-Arts, Paris, n. 97, p. 53-60, 1981; n. 98, p. 49-60, 1981.

AGULHON, Maurice. Un prof à Orsay. Le Débat, Paris, n. 44, p. 156-163, mars-mai 1987.

ALLARD, Sébastien. Le Louvre à l'époque romantique: les décors du palais (1815-1835). Paris: Musée du Louvre; Fage, 2006.

ALLIX, Grégoire. Un supermarché da la culture vendéenne. Le Monde, Paris, 11 août 2006.

ANCHEL, R. La commémoration des rois de France à Paris pendant la Restauration. Mémoires de la Société de l'histoire de Paris et de l'Ile de France, 4. Paris: Société de l'histoire da Paris et de l'Ile de France, 1924. p. 173-208.

ANGRAND, Pierre. Histoire des musées de province au XIXe siècle. Les Sables d'Olonne: Le Cercle d'or; Jean Huguet, 1984-1988. 9v.

AUDOUIN-ROUZEAU, Stéphane. La grande guerre prend fin aujourd'hui. Historia, Paris, n. 548, août 1992.

AUX GRANDS hommes, la patrie reconnaissante: 1867, le marquis de Laborde et le musée des Archives. In: Archives et Nations dans l'Europe du XIXème siècle. Paris: Ecole des Chartes, 2004. p. 215-233.

BACOT, Jean-Pierre; COQ, Christian. Travail de mémoire 1914-1998: une nécessité dans un siècle de violence. Paris:Autrement, 1999.

BACZKO, B. Une ville nommée Liberté. L'utopie et la ville. In: . Lumières de l'utopie. Paris: Payot, 1978. p. 65-69.

BAKHTINE, M. Esthétique et théorie du roman. Paris: Seuil, 1978.

BALZARRO,Anna. Le Vercors et la zone libre de l'Alto Tortonese: récits, mémoire, histoire.Thèse (Doctorat en Sciences Sociales) - École de Hautes Études en Sciences Sociales, Paris, janvier 2001.

BANN, S. The clotbing of Clio. A study of the representation of history in the XIXth Britain and France. Cambridge: Cambridge University Press, 1984.

BARCELLINI, Caroline. La commémoration de la Grande Guerre au musée de l'Armée (19141925). Guerres mondiales et conflits contemporains, Vincennes, n. 212, 2003. 
BARCELLINI, Serge. L'intervention de l'Etat dans les musées des guerres contemporaines. In: BOURSIER, Jean-Yves (Dir.). Musées de guerre et mémoriaux. Paris: Maison des sciences de l'homme, 2005.p 35-48.

BARTHES, Roland. Le discours de l'histoire. Social Science Information, v. 6, n. 4, p. 65-75, août 1967.

BECKER Annette. Musées ouverts, traces des guerres dans le paysage. In: JOLY, M.-H; COMPÈREMOREL,T. (Coord.). Des musées d'histoire pour l'avenir: actes du colloque de Péronne. Paris: Noesis, 1998.

BENOÎT, Isabelle. Politique de mémoire: les musées d'bistoire français et allemands, 19451995.Thèse (Doctorat) - Institut Universitaire Européen, Florence, 2001.

BENZAÏD, R. (Prés.). L'etbnologie de la France: Besoins et projets. Paris: La Documentation Française, 1980.

BERÇÉ, F. Le problème de la conservation in situ au XIXe siècle:le musée des Monuments français d'Alexandre Lenoir et le musée de Cluny. In: MinisTÈre DE lA CulTuRE. Entretiens du patrimoine 1992: Meubles et immeubles. Paris: Ministère de la Culture, 1993.p. 18-25.

BERNARD, Claudie. Le passé recomposé: le roman historique français du dix-neuvième siècle. Paris: Hachette, 1996.

BERNARD, Claudie. Démomification et remomification de l'histoire. Poétique, 88, p. 479-486, 1991.

BLETON-RUGET,Annie. Quand l'ethnographie de la France passait par la Bourgogne. Cabiers d'bistoire de la vigne et du vin, Dijon n. 6, p. 65-89, 2006.

BONNET, Jean-Claude. Naissance du Panthéon. Essai sur le culte des grands hommes. Paris: Fayard, 1998.

BOURSIER, Jean-Yves. Résistances et Résistants. Paris: L'Harmattan, 1997.

BRESC-BAUTIER, G. Tombeaux factices de l'abbatiale de Saint-Denis. In: BULLETIN de la Société nationale des antiquaires de France, Paris: SNAF, 1981. p. 115ss.

BROWER, Benjamin C. The Preserving Machine: The "New" Museum and Working through Trauma.The Musée Mémorial pour la Paix of Caen. History and Memory, Bloomington v. 11, n. 1 p. $77-103,999$.

CENTLIVRES, P.; FABRE, D.; ZONABEND, F. (Dir.). La fabrique des héros. Paris: Maison des sciences de l'homme, 1999.

CHAUDONNERET, Marie-Claude. Peinture et Histoire dans les années 1820-1830.In: L'Histoire au musée. Arles:Actes-Sud, 2004. p. 127-138.

CHAUDONNERET, Marie-Claude. Historicism and Heritage in the Louvre, 1820-1840: from the musée Charles X to the galerie d'Apollon. Art History, Londres, n. 14, p. 488-520, 1991.

CHEVALIER, Elsa. Le Musée de Bretagne: un musée face à son histoire. Rennes: PUR, 2001. 
COHEN, Cl.; HUBLIN, J.J. Boucher de Perthes. Les origines romantiques de la préhistoire. Paris: Belin, 1989.

CUISENIER, Jean. L’héritage de nos pères. Paris: La Martinière, 2006.

DARNIS, J.-M. Les monuments expiatoires à la mémoire de Louis XVI et de Marie-Antoinette. Paris: Union parisienne d'imprimerie, 1981.

DEMING, M. K. Le Panthéon révolutionnaire. In: LE PANTHÉON: symbole des révolutions, 1989, Paris, CNMHS. Catalogue (Dir. B. Bergdoll) Hôtel de Sully; Montréal, CCA: Picard, 1989. p. 97-150.

DIAS, N. Looking at objects: memory, knowledge in nineteenth-century ethnographic displays. In: ROBERTSON, G.; MASH, M.;TICKNER, L.; BIRD, J.; CURTIS, B.; PUTNAM, T. (Ed.). Travellers' Tales: narratives of home and displacement. London: Routledge, 1994. p. 164-176.

DONNAT, Olivier. Les Français face à la culture. Paris: La Découverte; Documentation française, 1994.

DUCLOS, J.-C. Pour des musées de l'homme et de la société. Le Débat, Paris, v. 70, p. 174-178, maiaoût, 1992.

DUCLOS, J.-C (dir.). D’Isère et du Maghreb: Mémoires d'immigrés. Grenoble: Musée dauphinois, 1999.

ENLART, Camille. Catalogue général du Musée de sculpture comparée au Palais Trocadéro. Paris:A. Picard, 1910.

FABRE, Daniel. Ancienneté, altérité, autochtonie. In: (Dir.). Domestiquer l'histoire: ethnologie des monuments historiques. Paris: Maison des sciences de l'homme, 2000.

FLEURY, E.Avant-propos. Terrain, Paris, n. 11, p. 5-7, 1988.

FRANCOIS, Emmanuelle. Les musées d'bistoire dans la Seconde Guerre mondiale: rapport au ministère de la Culture. Paris: Direction des musées de France-Ministère de la Culture, 1996.

GAEHTGENS, Thomas W. Versailles: de la résidence royale au musée historique. La galerie des batailles dans le musée historique de Louis-Philippe. Paris:Albin Michel, 1984.

. Le musée historique de Versailles. In: NORA, P. (Éd.). Les lieux de mémoire 2: La Nation, v. 3. Paris: Gallimard, 1986. p. 143-168.

GAUCHET, Marcel. Considérations sur l'histoire de France. In: NORA, P.(éd.). Les lieux de mémoire 2: La Nation. Paris: Gallimard, 1986. p. 317-375.

GEORGES, Nicolas. Dans le rapport la création d'un centre national de l'bistorie et des cultures de l'immigration, par Driss Elyazami et Rémy Scwartz. Paris, 2001.

GOSSMAN, Lionel. Medievalism and the Ideologies of Enlightenment:The world and work of La Curne de Sainte Palaye. Baltimore: Johns Hopkins University Press, 1968.

Between History and Literature. Baltimore: Johns Hopkins University Press, 1990. 
GRAN-AYMERICH, Eve. Naissance de l'archéologie moderne, 1798-1945. Paris: Centre national de la recherche scientifique, 1998.

HARTOG, François. L'oeil de l'historien et la voix de l'histoire.In: Le genre bumain. Paris: Seuil, 1996.p. 55-69.

HERZFELD, M. Cultural intimacy. Social poetics in the Nation-State. Londres: Routledge, 1997.

HUGO, Victor. Discours à la inauguration triomphale du musée de Louis-Philippe le 10 juin 1837. In: Choses vues 1830-1846, Paris: Gallimard Folio, 1972.

HUTTON, Patrick. The Art of Memory Reconceived: from Rhetoric to Psychoanalysis.Journal of the History of Ideas, v. 48, n. 3, p. 371-392, July-Sept., 1987.

IDJÉRAOUI, L.; DAVALLON, J. Le témoignage peut-être un objet de musée? In: COLLOQUE INTERNATIONAL: Recherches récentes en sciences de l'information, Toulouse, 21-22 mars 2002, Actes. Toulouse:Adbs, 2002.

IFRI, Philippe; GUENEAU, Maurice; MAUBANT, Philippe (Dir.). Ecomusées et musées de sociétés: dire l'histoire et gérer la mémoire au présent. Pour, n. 153, p. 147-153, mars 1997.

JAKOBSON, R. Du réalisme en art . In: Questions de poétique. Paris: Seuil, 1973.

JOLY, M.-H. (Dir.). Guide des musées et collections d'bistoire en France. Marseille:Association internationale des musées d'histoire, 1996.

JOLY, M.-H;. COMPÈRE-MOREL,T. (Coord.). Des musées d'histoire pour l'avenir: actes du colloque de l'Historial de Péronne. Paris: Noesis, 1998.

KAPLAN, Fl. E. S.Museums and the making of "ourselves". The role of objects in national identity. Londres: Leicester University Press, 1996.

KAVANAGH, Gaynor. History Curatorship. Leicester: Leicester University Press, 1990.

LA JEUNESSE DES MUSÉES: les musées de france au XIXe siècle, 1994, Paris, Musée d'Orsay. Catalogue (Dir.Chantal Georgel). Paris: Réunion des musées nationaux, 1994.

LAZIER, Isabelle; DESVALLÉES, André (Réd.). Ecomusées en France: Premières Rencontres nationales des écomusées, L'Isle-d'Abeau, 13 et 14 novembre 1986. Grenoble:Agence régionale d'ethnologie; Rhône-Alpes: Ecomusée Nord-Dauphiné, 1987.

LEBLANC, Henri. Catalogue raisonné des estampes, originaux, affiches illustrés, imageris, vignettes, cartes postales, médailles, bons de monnaies, timbres etc. In: La Grande Guerre: Iconograpie, bibliographie, documents divers, I. Paris: Emile Paul, 1916.

LE GOFF, J. Histoire et Mémoire. Paris: Gallimard, $1977 a$.

. Préface. In: BLOCH, Marc. Apologie pour l’histoire ou Métier d'bistorien. Paris:A. Colin, $1997 b$.

LENNON, J. J.; FOLEY, M. (Ed.). Dark Tourism: the attraction of death and disaster. Londres: Continuum, 2000. 
LES MUSÉES EN EUROPE À LA VEILLE DE L'OUVERTURE DU LOUVRE, 1995, Paris, Musée du Louvre. Actes. (Dir. Edouard Pommier). Paris: Klincksieck; Musée du Louvre, 1995.

LE PANTHÉON: symbole des révolutions, 1989, Paris, CNMHS. Catalogue (Dir. B. Bergdoll) Hôtel de Sully; Montréal, CCA: Picard, 1989.

LÉVI-STRAUSS, Claude. La pensée sauvage. Paris: Plon, 1962.

LÖWENTHAL, David. The past is a foreign country. Cambridge: Cambridge University Press, 1985.

MAROT, P. Les origines d'un musée d'antiquités nationales. In: Mémoires de la société des antiquaires de France, IV. Paris: SNAF, 1981, p. 259-327.

MARTIN, Jean-Clément; SUAUD, Charles. Le Puy du Fou, en Vendée: l'histoire mise en scène. Paris: L'Harmattan, 1996.

MCCLELLAN,A. La série des grands hommes de la France du comte d'Angiviller et la politique des Parlements. In: CLODION ET LA SCULPTURE FRANÇAISE DE LA FIN DU XVIIIÈME SIÈCLE, Paris, Musée du Louvre, 20-21 mars 1992. Actes.... Paris: Musée du Louvre, 1993.

MELLON, Stanley. The political uses of bistory: a study of historians in the french restoration. Stanford, CA: Stanford U.P., 1958.

MUSÉE des Monuments Français. Catalogue du Musée des Monuments Français. Paris: MMF, 1810 .

PINATEL, Ch. Origines de la collection des moulages d'antiques de l'Ecole nationale des BeauxArts de Paris, aujourd'hui à Versailles. In: LAURENS, M.-F; POMIAN, K. (Éd.). L'Anticomanie: La collection d'antiquités aux XVIIIème et XIXème siècles. Paris: Editions de l'Ecole des Hautes Etudes en Sciences Sociales, 1992. p. 307-325.

QUETEL, Claude. Un Mémorial pour la paix. Caen: Regard, 1992.

RAPHAËL, F; HERBERICH-MARX, G. Le musée, provocation de la mémoire. Ethnologie française, Paris, v. 17, n. 1, p. 87-95, 1987.

REGARDS sur l'actualité culturelle du Musée de l'Armé. L'echo du dome, Paris, n. 10, sept. 2007.

RICHARD, Nathalie. La préhistoire au quotidien. La pratique de l'archéologie préhistorique au XIXème siècle, d'après les correspondances réunies au Musée des Antiquités Nationales de SaintGermain-en-Laye. Gradbiva, Paris, n. 9, p. 77-94, 1991.

RICOEUR, Paul. L'écriture de l'histoire et la représentation du passé. Annales HSS, Paris, 22, 4, p. 731-747, 2001.

SEGALEN, Martine (Dir.).L'autre et le semblable.Paris: Centre national de la recherche scientifique, 1989.

Vie d'un musée, 1937-2005. Paris: Stock, 2005. 
SHERMAN, Daniel J.Art History and Art Politics: the Museum According to Orsay.Oxford Art Journal, Oxford, v. 13, n. 2, p. 55-67, 1990.

. Objects of Memory: History and Narrative in French War Museums.French Historical Studies, v. 19, n. 1, p. 49-74, 1995.

SOUCHAL, Fr. Situation de la sculpture en France en 1778. In: Dix-Huitième Siècle, Paris: PUF, 11. 1979. p. 117-121.

TOLLEBEEK, Jo. Seeing the Past with the Mind's Eye: the Consecration of the Romantic Historian. Clio, v. 29, n. 2, p. 167ss., 2000.

VERGNON, Gilles. Le mémorial de Vassieux, un mémorial hors sol? In: BOURSIER, Jean-Yves (Dir.). Muséees de guerre et mémoriaux. Paris: Maison des sciences de l'homme, 2005. p. 155-162.

WAHNICH, Sophie. Trois musées de guerre du XXème siècle. In: BOURSIER, Jean-Yves (Dir.). Muséees de guerre et mémoriaux. Paris: Maison des sciences de l'homme, 2005. p. 80-81.

WEBER, Florence. Politiques du folklore en France 1930-1960. In: MINISTÈRE DE LA CULTURE. Pour une histoire des politiques du patrimoine. Paris: Comité d'histoire du ministère de la culture, 2003. p. 269-300.

WHITMARSH,Andrew."We will remember them": memory and commemoration in war museums. Journal of Conservation and Museum Studies, Londres, v. 7, p. 1-15, nov. 2001.

YOUNG, James E. The texture of memory: Holocaust memorials and meaning. New Haven:Yale University Press, 1993a.

1993b.

Ecrire le monument: site, histoire, critique. Annales ESC, Paris, v. 48, n. 3, p. 729-743, juin,

Artigo apresentado em 4/2007. Aprovado em 5/2007. 